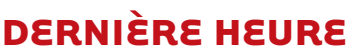

\section{Réhabilitation de la dystrophine humaine dans le modèle scid/mdx après greffe de cellules souches de patients DMD corrigées par saut d'exon}

Rachid Benchaouir, Mirella Meregalli, Andrea Farini, Giuseppe D’Antona, Marzia Belicchi, Aurélie Goyenvalle, Maurizio Battistelli, Nereo Bresolin, Roberto Bottinelli, Luis Garcia, Yvan Torrente
Dystrophine et mutations du gène dans la DMD

$>$ La myopathie de Duchenne affecte principalement les garçons (1 sur 3500 ). Elle est due à des mutations portant sur le gène de la dystrophine, localisé sur le chromosome X. C'est le plus grand gène connu, il est formé de 79 exons dispersés sur un segment de près de 2,5 millions de nucléotides. Comme pour la plupart des maladies génétiques, le spectre des mutations est varié, mais ici il existe

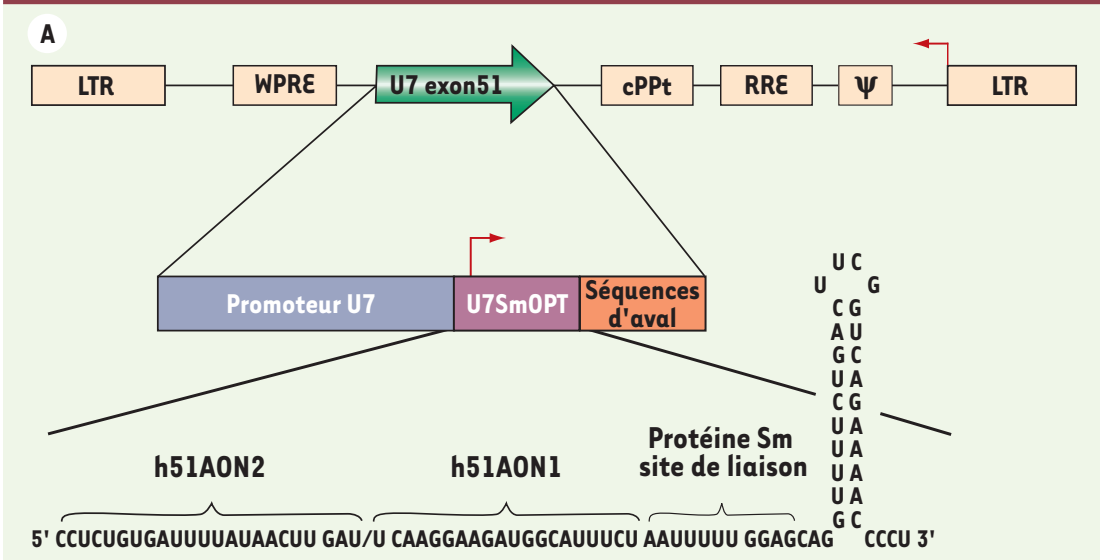

B

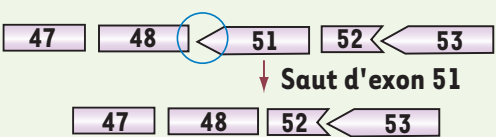

Figure 1. A. Schéma du vecteur lentiviral codant le snARN U7opt-ex51. Le U7snARN transformé en U7opt-ex51 spécifique de l'exon 51 du gène de la dystrophine humaine est cloné entre le WPRE et le cPPT. Le petit ARN non codant modifié est placé sous le contrôle de son propre promoteur et de ses régions 3' régulatrices. LTR : long terminal repeats; Psi : séquence signal d'encapsidation ; RRE : Rev-responsive element; $\mathrm{CPPT}$ : central polypurine tract ; WPRE : woodchuck hepatitis virus responsive element. B. Représentation du phasage exonique au voisinage de la délétion $\Delta 49-50$ et stratégie de réhabilitation par élimination de l'exon 51.
R. Benchaouir, M. Meregalli, A. Farini, M. Belicchi, M. Battistelli, N. Bresolin, y. Torrente: Stem Cell Laboratory, Department of Neurological Sciences, Fondazione IRCCS Ospedale Maggiore Policlinico, Centro Dino Ferrari, University of Milan, via F. Sforza 35, 20122 Milan, Italie. y. Torrente : UNISTEM,

Centro Interdipartimentale di Ricerca sulle Cellule Staminali, University of Milan, via Balzaretti 9, 20133 Milan, Italie.

G. D’Antona, R. Bottinelli :

Department of Experimental Medicine, Human Physiology Unit, University of Pavia, via Forlanini 6, 27100 Pavia, Italie.

L. Garcia : UPMC-Inserm UMR S 787, Institut de Myologie,

Faculté de Médecine Pierre et Marie Curie, 105, boulevard de l'Hôpital, 75634, Paris Cedex 13, France. yvan.torrente@unimi.it luis.garcia@chups.jussieu.fr

un fait remarquable: deux tiers des mutations sont des délétions emportant un ou plusieurs exons. Les effets pathogènes de ces délétions dépendent des répercussions sur le produit final: la dystrophine. Soit le cadre de lecture de l'ARNm muté est aboli, et le tableau clinique est celui d'une dystrophinopathie sévère (DMD, dystrophie musculaire de Duchenne), soit le cadre de lecture est préservé, une dystrophine amputée peut être synthétisée en quantités plus ou moins importantes et le phénotype résultant est d'autant plus atténué (BMD, dystrophie musculaire de Becker). Il est important de souligner que des individus «asymptomatiques » porteurs de larges délétions ont été fortuitement identifiés. Ces sujets ne présentent pas de signes cliniques particuliers si ce n'est un taux de créatine kinase musculaire un peu plus élevé que la normale. La tolérance d'amputations partielles «en phase » s'explique par la structure modulaire de la dystrophine. Elle présente un module d'interaction avec l'actine à 
son extrémité amino-terminale. Sa partie centrale est constituée de 24 domaines répétés de type spectrine, suivie d'une région riche en cystéine permettant son ancrage à un complexe multi-protéique membranaire en interaction avec la matrice extracellulaire. Elle se termine enfin par un domaine carboxy-terminal associant des protéines de signalisation. Le domaine riche en cystéine est de loin le plus important et sa perte anéantit la fonction de la dystrophine. La fonction exacte de la dystrophine reste encore énigmatique. Sans elle, les tissus musculaires (squelettiques, lisses et cardiaques) sont fragilisés et se détériorent lorsqu'ils sont soumis à l'exercice naturel. Le fait qu'elle soit la clé de voûte d'un continuum protéique liant le cytosquelette à la lame basale musculaire suggère une implication dans la résistance mécanique des fibres. Un rôle purement mécanique est cependant peu plausible du fait de sa relative inabondance dans les muscles sains. Une hypothèse plus
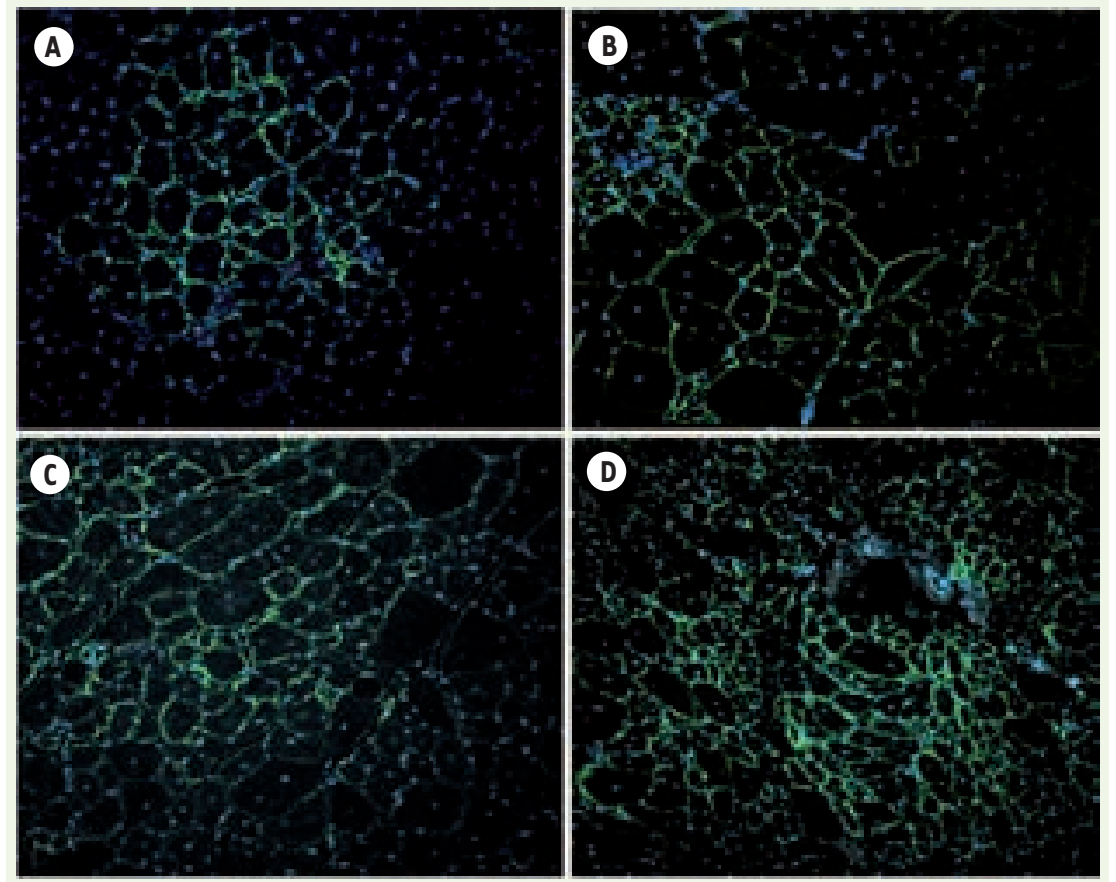

Figure 2. Immunomarquage de la dystrophine avec l'anticorps monoclonal de souris NCL-Dys3 (Novocastra). Sections de muscles (tibialis antérieur) de souris scid/mdx, 3 semaines et 6 semaines après injection intramusculaire de cellules $C D 133^{+}$transduites provenant d'un patient $\Delta 49$ 50 (origine sanguine $\boldsymbol{A}$ et $\boldsymbol{B}$; origine musculaire $\boldsymbol{C}$ et $\boldsymbol{D}$ ). de transplantations de myoblastes se sont souvent révélées décevantes. Les myoblastes greffés migrent peu et, s'il s'agit de greffons allogéniques, sont rapidement rejetés en l'absence d'immunosuppression. Au cours de la dernière décennie, de nombreux travaux ont révélé des filiations cellulaires inattendues entre différents tissus de l'organisme: des cellules souches d'origine hématopoïétique ou vasculaire peuvent occasionnellement participer à la formation des fibres musculaires en régénération. C'est le cas des cellules $\mathrm{CD}_{133^{+}}$que l'on peut isoler à partir de biopsies musculaires ou à partir de prélèvements sanguins [2]. Le potentiel myoendothélial de ces cellules a été confirmé ex vivo et in vivo, et une première étude clinique visant à vérifier chez des patients DMD l'innocuité d'une administration locale de cellules $\mathrm{CD} 133^{+}$autologues a été récemment publiée [3]. Notre objectif était maintenant de corriger les cellules des patients avant réinjection. À cette fin, nous avons choisi d'équiper les cellules $\mathrm{CD} 33^{+}$fraîchement isolées d'un système de saut d'exon thérapeutique (SET). Le SET consiste à restaurer un cadre de lecture opérationnel d'un transcrit muté en éliminant un ou plusieurs exons de I'ARNm. Cette opération est rendue possible par l'usage de séquences antisens masquant les motifs de définition du ou des exons bornant la mutation lors de la réaction d'épissage de l'ARN pré-messager. Dans la majorité des cas, l'ARNm ainsi réhabilité permet la synthèse d'une dystrophine certes tronquée mais fonctionnelle. Les séquences antisens peuvent être des oligonucléotides synthétiques qu'il faut réapprovisionner régulièrement, ou bien être camouflées dans un transgène correspondant à un petit ARN non codant (snARN U7 ou U1) modifié ou vectorisé, ce qui assure une production permanente de l'antisens [4, 5]. Pour cette étude, nous avons cherché à effectuer un SદT dans des cellules de patients DMD génotypés présentant une délétion des exons 49 et 50 (qui décale le cadre de lecture).

Le vecteur produisant le SET est une cassette d'expression d'un snARN U7 modifié 

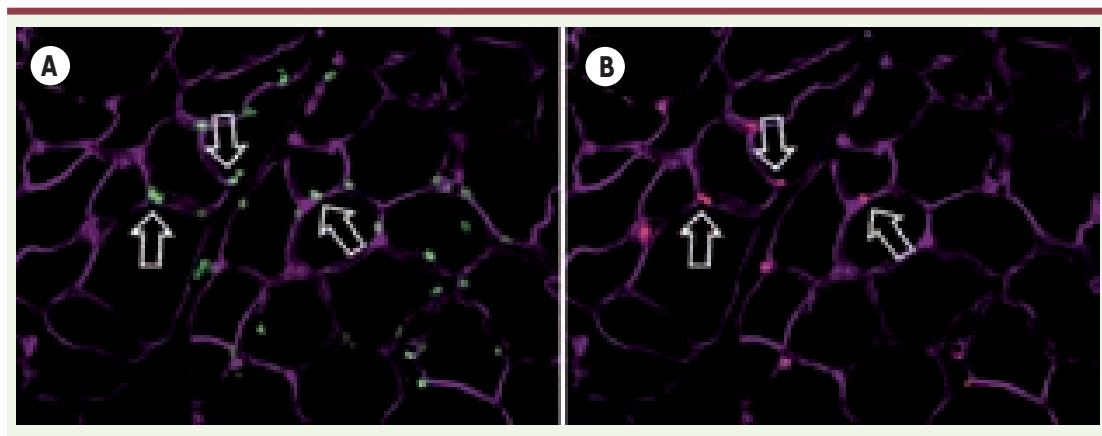

Figure 3. Localisation en position satellite des cellules $\mathrm{CD}_{133^{+}}$greffées. $A$. Immuno-marquage lamine $A / C$ humaine (vert). B. Immuno-marquage $M$-Cadhérine (rouge). Les lames basales sont marquées en magenta.

portant des séquences antisens empêchant l'inclusion dans l'ARNm de l'exon 51. Cette stratégie s'était déjà montrée très efficace in vivo chez la souris $m d x$ à l'aide d'une cassette vectorisée dans un virus AAV [4, 5]. Mais ce vecteur étant incapable de transduire des cellules ex vivo, nous avons intégré la cassette thérapeutique dans un vecteur lentiviral capable de transduire et de s'intégrer dans le génome des cellules $\mathrm{CD}_{133^{+}}$. La construction est décrite dans la Figure $1 A$, et le saut d'exon correcteur recherché, et effectivement obtenu, est représenté dans la Figure $1 B$.

L'évaluation in vivo a été réalisée en réinjectant dans le modèle de souris scid/ $m d x$ les cellules $\mathrm{CD} 133^{+}$du patient DMD $\triangle 49-50$ préalablement transduites ex vivo. Ces souris sont à la fois dépourvues de dystrophine et immunodéficientes, donc tolérantes vis-à-vis des xénogreffés. Dès trois semaines après injection intramusculaire, de nombreuses fibres expriment au niveau du sarcolemme une dystrophine humaine réhabilitée au niveau du sarcolemme. Après six semaines, ce nombre est doublé pour les $\mathrm{CD} 133^{+}$originaires du sang et plus que quadruplé pour les cellules isolées à partir du tissu musculaire (Figure 2). Parallèlement, on observe la réapparition à la membrane des protéines du complexe des glycoprotéines associées à la dystrophine. Enfin, l'identification des noyaux humains par un anticorps anti-lamine $A / C$ permet d'identifier de nombreuses cellules greffées en position satellitaire des fibres musculaires, à l'instar des précurseurs musculaires canoniques (Figure 3). Cette observation suggère que ce mode opératoire a permis d'implanter un réservoir de progéniteurs musculaires corrigés qui pourra être mobilisé à la demande au rythme des nécroses-régénérations avoisinantes. Enfin, les cellules $\mathrm{CD} 133^{+}$transduites ont également été administrées via l'artère fémorale. Ici encore, des foyers de fibres exprimant la dystrophine humaine ont pu être identifiés, en particulier dans le muscle tibial antérieur, démontrant la capacité d'extravasation de ces cellules. Le fait que le nombre de fibres positives semble inférieur à celui obtenu par injection intramusculaire ne doit pas minorer l'importance du résultat. Ici les cellules greffées ont été disséminées à travers l'ensemble du système vasculaire et se sont vraisemblablement nichées à bas bruit dans de nombreux muscles. Les tests fonctionnels semblent corroborer cette hypothèse.

\section{Perspectives thérapeutiques}

Ces résultats laissent entrevoir la possibilité d'une thérapie cellulaire pour DMD par transfert de cellules souches autologues préalablement équipées d'un dispositif moléculaire assurant la réhabilitation par saut d'exon de l'ARN messager de la dystrophine. Loin d'être en concurrence avec les approches de thérapie génique directe (injection de vecteurs thérapeutiques) ou pharmacogénétiques (AON synthétiques), cette approche se veut complémentaire et vise à stimuler/renforcer le potentiel régénératif chez des patients déjà installés dans le processus myopathique et présentant un tissu musculaire très endommagé. L'accessibilité des cellules CD133+ (isolées à partir du sang circulant) et leur origine autologue simplifient d'autant le problème du rejet de greffe tout en autorisant des traitements récurrents. Comme toute approche de thérapie génique, il reste encore à étudier l'impact de l'insertion du génome du vecteur thérapeutique sur le devenir des cellules CD133+. Nous nous attachons en particulier à évaluer l'éventualité d'événements tumorigènes par transplantation des cellules transduites dans la souris NOD/SCID. $\diamond$

Restoration of human dystrophin following transplantation of exon-skipping-engineered DMD patient stem cells into dystrophic mice

\section{REMERCIEMENTS}

Ce travail a été soutenu par : AMM (Association Monégasque contre les Myopathies); AFM (Association Françaisecontreles Myopathies); DPPF (Duchenne Parent Project France); Associazione La Nostra Famiglia Fondo DMD Gli Amici di Emanuele; Associazione Amici del Centro Dino Ferrari; CNRS et Financement Européen (MyoAMP-FP6).

\section{RÉFÉRENCES}

1. Benchaouir R, Meregalli M, Farini A, D’Antona G, Belicchi M, Goyenvalle A, Battistelli M, Bresolin N, Bottinelli R, Garcia L, Torrente Y. Restoration of human dystrophin following transplantation of exonskipping-engineered DMD patient stem cells into dystrophic mice. Cell Stem Cell 2007 ; 1 : 646-57.

2. Torrente $Y$, Belicchi M, Sampaolesi M, et al. Human circulating $\mathrm{ACl} 33(+)$ stem cells restore dystrophin expression and ameliorate function in dystrophic skeletal muscle. J Clin Invest 2004 ; 114 : 182-95.

3. Torrente $Y$, Belicchi M, Marchesi C, et al. Autologous transplantation of muscle-derived $\mathrm{CD}_{133^{+}}$stem cells in Duchenne muscle patients. Cell Transplant $2007 ; 16: 563-77$.

4. Goyenvalle A, Vulin A, Fougerousse F, Leturcq F, Kaplan JC, Garcia L, Danos O. Rescue of dystrophic muscle through U7 snRNA-mediated exon skipping. Science 2004 ; 306 : 1796-9.

5. Goyenvalle A, Vulin A, Fougerousse F, Leturcq F, Kaplan JC, Garcia L, Danos 0. Le saut d'exon thérapeutique : un espoir pour les dystrophinopathies. Med Sci (Paris) 2004 ; $20: 1163-5$.

\section{TIRÉS À PART}

L. Garcia et Y. Torrente 